Persuasive Technology

\title{
An Alternative Aid For Children With Selective Mutism \\ Through Collaborative Healthware
}

by

Nuria Gonzalez

\author{
A Major Research Paper \\ presented to Ryerson University \\ in partial fulfillment of the requirements for the degree of \\ Masters in Digital Media \\ in the Program of Digital Media \\ Toronto, Ontario, Canada, 2017 \\ (C) Nuria Gonzalez, 2017
}




\section{Author's Declaration}

I hereby declare that I am the sole author of this MRP. This is a true copy of the MRP, including any required final revisions.

I authorize Ryerson University to lend this MRP to other institutions or individuals for the purpose of scholarly research.

I further authorize Ryerson University to reproduce this MRP by photocopying or by other means, in total or in part, at the request of other institutions or individuals for the purpose of scholarly research.

I understand that my MRP may be made electronically available to the public 
An Alternative Aid For Children With Selective Mutism Through Collaborative Healthware

Master of Digital Media, 2017

Nuria Gonzalez

Master of Digital Media, Ryerson University

\begin{abstract}
This major research paper investigates the understanding of the role of persuasive technology in the implementation of alternative digital platforms to support mental health treatments in children; specifically those related to selective mutism. It analyzes the effectiveness in the behavioural change by integrating trigger functions into a wearable device. At the same time, this paper illustrates how this technology can help caregivers manage treatments using collaborative healthware.
\end{abstract}




\section{Acknowledgements}

This paper would not be possible without the support and guidance of my supervisor

Dr. Alexander Ferworn. His constant encouragement and feedback gave me the confidence and knowledge to complete this paper. I would also like to thank Professor Michael Carter for agreeing to be my second reader and for his unlimited patience. Professor Carter made himself available throughout the time of the program and was always present when needed. Moreover, he always gave me invaluable advice, not just professionally, but personally. I would like to thank my amazing husband, Ignacio, for his unconditional love and support throughout this year. I know it was difficult to be a mom and a dad at the same time, thank you for your love and patience. This is an important milestone for us, not just me, and I will be eternally grateful for you being by my side and sharing this exciting year with me. Thank you for all the meals you made without me and the errands you ran for us, and of course the love you have always given us. This is for Nicolas and Cecilia. To all my professors in the MDM program, every one of them contributed in one way or another to the completion of this paper. Thank you Rob Blain, Wojtek Kawczynski, Naimul Khan, Richard Lachman, Paul Moore, Ramona Pringle, Kaamran Raahemifar and Kathryn Woodcock. Thank you to my 4.0 cohort, your friendship, support and talent is sincerely treasured. Specially Soo Mahabir for her invaluable help and honest friendship, I was always looking forward to Saturdays. 


\section{To Cecilia}


Abstract

Acknowledgements iv

Dedication v v

List of Figures, Charts and Tables viii

$\begin{array}{ll}\text { Chapter 1: Introduction } & 1\end{array}$

$\begin{array}{ll}\text { Chapter 2: Literature Survey } & 4\end{array}$

$\begin{array}{ll}\text { Persuasive Technology } & 4\end{array}$

Definition of Persuasive Technology 4

i) Behaviour Change Support Systems

ii) The Fogg Persuasive Model (FBM) 9

Personalizing Persuasive Technology 12

Persuasive Technology in Educational Settings $\quad 14$

Anxiety Disorder: Selective Mutism $\quad 19$

Definition of Selective Mutism (SM) 19

Characteristics and Social Challenges of Children Diagnosed with SM 19

$\begin{array}{ll}\text { Medical and Psychological Interventions } & 21\end{array}$

Healthware: Digital Healthcare and Innovation 23

Collaborative Healthware $\quad 24$

Advantages and Challenges of Collaborative Healthware 25

Healthware as aid on treatment for children $\quad 27$

Chapter 3: Analysis, Methodology and Implementation 30

Analysis: Selective Mutism and Collaborative Healthware 31

Methodology: Behaviour change by implementing the Fogg Method, resulting in teaching anxiety coping mechanisms to selectively mute children 32 Implementation $\quad 35$

Chapter 4: Case Studies $\quad 38$

Reveal, by Awake Labs $\quad 31$

The HANDS Project: Helping Autism Diagnosed young people Navigate and Develop Socially 41

Aparito $\quad 42$

Habitica: Habit RPG (Role Player Game) 43 
Chapter 5: Conclusion

References 


\section{List of Figures, Charts and Tables}

Diagram 1: Phases in Persuasive System Development.

Source: Oinas-Kukkonen, Harri and Harjumaa, Marja (2009) Persuasive Systems

Design: Key Issues, Process Model, and System Features

\section{Chart: Fogg Behavior Model}

Source: Fogg, B. (2009). A Behavior Model For Persuasive Design

Table: Applying Persuasive Design Principles in Instructional Design

Source: Mintz, J., and Aagaard, M. (2012). The application of persuasive technology to educational settings.

Diagram 2: Conceptual Framework to Describe the Application of Technologies in Clinical Care and Research with Children with Chronic Conditions

Source: Siarkowski Amer, K. (2006). Innovations in Pediatric Health Care Technology: A Multidisciplinary Conceptual Framework for Using and Evaluating Information Systems. 


\section{Chapter 1: Introduction}

The immersion of digital technologies in the healthcare industry in the last decades, has helped in the advancement of medical research and treatment in areas such as

Mental Health. Applying these technologies in the field of mental health research has allowed us to gain a much better understanding of disorders such autism and anxiety disorder, and thereby develop more effective supports for treatments to patients.

Technologies, such as machine learning and data analytics, have been applied to the study of behavioural patterns in people within the autism spectrum. As a result, this kind of research has started to permeate in other areas, such as anxiety disorders. This paper is rooted in and contributes further to the existing arguments within the field which state that combining these technologies would result in the better understanding of mental patterns and behavioural change. Moreover, researchers in behavioural change designed based technology, have proposed and implemented methods, such as persuasive technology, which make the development of behavioural change frameworks possible. These frameworks apply interventions such as motivation, abilities and triggers in order to generate such changes. These methods have also been applied in educational settings as a base learning tool for children within the autism spectrum. 
Based on the growing advances in persuasive technology, as well as in the research done on collaborative healthware, there has been an increase in the creation of supporting tools in the treatment of anxiety disorders. This approach could benefit the outcome of treatment of children diagnosed with Selective Mutism (SM). These benefits could reach beyond the patient and their family, to the medical institutions as well.

As this paper focuses on treatments for SM, it is essential to first define the disorder. Selective Mutism is a rare anxiety disorder in which children withhold speech in social situations, for example at school or with playmates (despite having the physical capacity to do so), but they speak normally on other situations (Baldwin and Cline, 1991). Some of the characteristics of children with SM are shyness, fear of social embarrassment, withdrawal, social isolation, clinging behaviour, temper tantrums, control issues, and oppositional behaviour (Hungerford, Edwards, and lantosca, 2003; Krysanski, 2003). There is a common misconception that SM is a condition that the child outgrows. Therefore, it is essential to understand this long term condition well in order to develop and implement successful treatments.

As patient treatment becomes more inclusive within healthcare, the implementation of collaborative healthware becomes more accessible to patients. Collaborative healthware is the application of information and communication technologies 
designed to enhance decision-making and communication between providers, patients, and their families. This will benefit the communication between everyone involved with the child, as well as helping in the decision-making during the course of the treatment.

In conclusion, this major research paper investigates the understanding of the role of persuasive technology in the implementation of alternative digital platforms to support mental health treatments in children, specifically those related to SM. Moreover, it will analyze the effectiveness of adding timely trigger functions integrated into a wearable device in creating the desired behavioural change. At the same time, it aims to understand how this technology can help caregivers to manage treatment better by applying it to collaborative healthware. This paper will argue that the implementation of persuasive technology as an alternative aid for treating children with Selective Mutism will benefit not just the child in their treatment, but also will help their family, physicians and teachers to make more informed decisions in terms of course of treatment. 


\section{Chapter 2: Literature Survey}

\section{Persuasive Technology}

\section{Definition of Persuasive Technology}

Persuasive Technology (PT) is a technology designed to change attitudes and behaviours of users through persuasions, and to reinforce, change, or shape attitudes without using intimidation or deception. The role of persuasive technology is not to deliver a third party intervention where the user follows instructions with minimal understanding. Instead, it helps users to reflect and learn about their behaviour change, obstacles, and outcomes (Fogg, 2009).

In understanding the way PT functions, it is important to note that computers do not have intentions on their own but rather are designed, programmed and distributed with an intent to affect and change someone's behaviour. There have been studies that suggest PT uses similar patterns of interaction to human-to-human interactions (Fogg, 2009). Moreover, this technology is designed to reinforce and/or change a desired behaviour.

Social reactions to certain types of computing systems seem natural and automatic due to hardwired human reactions, especially to things that are relatable to a familiar behaviour. At some level, a person cannot control these social responses; they are instinctive rather than rational (Fogg, 2003). Moreover, persuasion has significant implications in computer system design due to the known fact that people respond 
socially to computers. Persuasive technology designers must understand and consider a number of socially related factors, such as attractiveness, opinions and attitudes, lifestyle, background and membership, in designing PT (Fogg, 2009).

Attractiveness also has a significant impact in social influence. The more visually attractive the presentation of a product, the more influence it will have on the target. This attraction is relative to the person's views of aesthetics, hence, PT designers need to understand their target audience when creating a product (Fogg, 2009). These aspects, which can be evaluated in considering whether a product attractive, can vary according to culture, age, gender as well as from individual to individual. Context is very important in determining which elements are considered attractive to the user/target audience.

Persuasion is most powerfully accepted when there is similarity. The principle of similarity suggests that people that seem alike (in personality, preferences and attributes) have a bigger influence on one another. Similarities between people emerge in opinions, attitudes, personal traits, lifestyles, backgrounds and memberships to different groups (Fogg, 2003). Even trivial similarities could potentially have a big influence on the user, for example: liking the same television show or rooting for the same hockey team. 
According to Long, Spurlock, Fjeldsoe, Marshall, Miller, Noell, (2013), another form of influence emerges through language and praise. While language is very important in communicating the message that the computer wants to convey, praise is also a highly persuasive method. Studies on the effect of praise in various situations have clearly shown that it has a positive impact. Some of the results in a research study conducted by Fogg in 2009 demonstrated that people responded more positively to praise, arguing that they felt better about themselves and that they had a better performance, were in better mood, and found the interaction engaging. This engagement is also seen in the reciprocity people feel with technology. People often feel the need to reciprocate when computing technology has done, what they perceive of as, a favour to them. This rule of reciprocity is based on a social dynamic that should be applied when designing persuasive technology.

In order to demonstrate the validity of PT, a Behaviour Change Support System (BCSS) should be applied. The main research refers not only to the human-computer interaction but also the computer-mediated communication, including methodologies, processes and tools to develop systems and ways for analyzing the social organizational and impact of the technology in the end-user (Oinas-Kukkonen, Harri and Harjumaa, Marja, 2009). 


\section{i) Behaviour Change Support Systems}

The BCSS is an information system designed to alter or reinforce attitudes, behaviours, or an act of complying without using deception, coercion or inducements (Oinas-Kukkonen, Harri and Harjumaa, Marja, 2009). In order to apply BCSS effectively there must be a stakeholder who has the intention of influencing someone's behaviour.

Postulations need to be considered when applying BCSS. One postulation is that information technology is never neutral, which means it is always influencing attitudes in one way or another. This persuasion is constantly applied in the same way teachers persuade students during class. This is not considered to be "wrong", although there are some ethical considerations to be made. There is a need to consider different factors, such as stakeholder and user goals, and the process and time that this might take (Oinas-Kukkonen, Harri and Harjumaa, Marja, 2009). Another consideration is predictability and consistency. If this system supports making a commitment and looks consistent in its response, users will be more likely to be persuaded by it. This can be implemented, for example, by offering an easy way to send a text message or email to one's relatives, friends and/or colleagues (Oinas-Kukkonen, Harri and Harjuaa, Marja, 2009). 
When developing persuasive systems, it's important to consider the following three steps, which are illustrated in the diagram below (Oinas-Kukkonen, Harri and Harjumaa, Marja, 2009):

1. It is fundamental to understand the issues behind implementing a persuasive system before implementing it. And it is imperative to analyze and understand the implications of this behaviour change before designing a system.

2. The second phase consists of recognizing and analyzing the intent, events and strategies for the use of this system.

3. The third phase evaluates set system. A new information system might be designed to achieve the desired behaviour change.

Many design aspects in developing BCSSs are general software design issues rather than those specific to BCSSs only. These include: usefulness, ease of use, ease of access, quality of information, simplicity, convenience, attractiveness, lack of errors, responsiveness, high overall positive user experience, and user loyalty (Oinas-Kukkonen, Harri and Harjumaa, Marja 2009). 

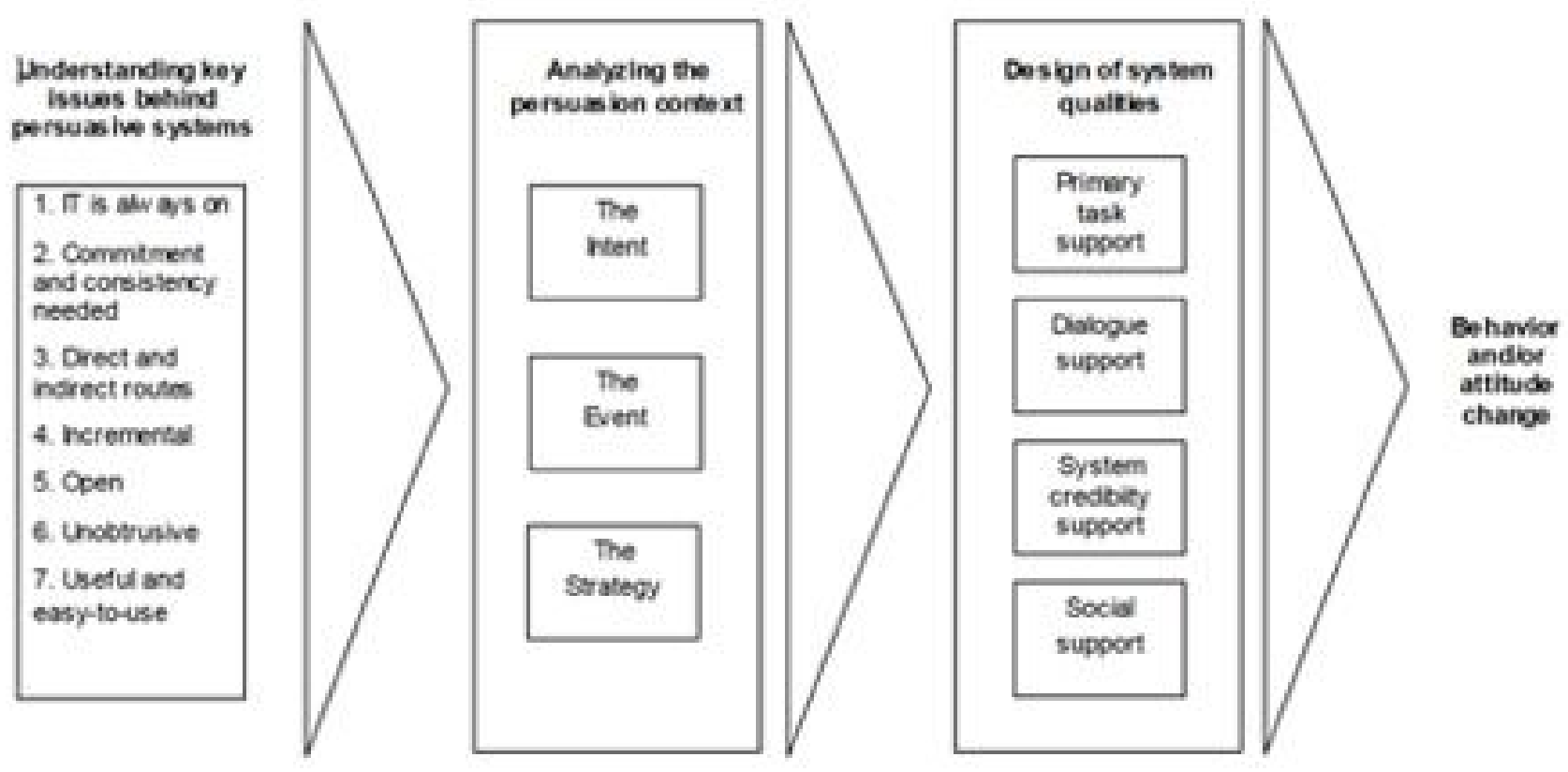

Diagram 1: Phases in Persuasive System Development. Source: Oinas-Kukkonen, Harri and Harjumaa, Marja (2009) Persuasive Systems Design: Key Issues, Process Model, and System Features

Persuasive Technology is directly related to active assistance technology, which is defined as any technology that involves ongoing automated processing of information relating to health and behaviour change as the user interacts with the technology (Fogg, 2003) The processing of unbiased information by this technology is favourable to self-monitoring and correction of inaccurate health perception, both of which are required in the persuasion model.

\section{ii) The Fogg Persuasive Model (FBM)}

The FBM is based on the fundamentals of learning to automate behaviour change. In order to encode change in behaviour experiences there should be a clear understanding of human psychology, especially the motivation and drive of human 
behaviour (Fogg 2009). For the FBM to be implemented, the user must have the three following attributions: he/she should have motivation to act, the ability to

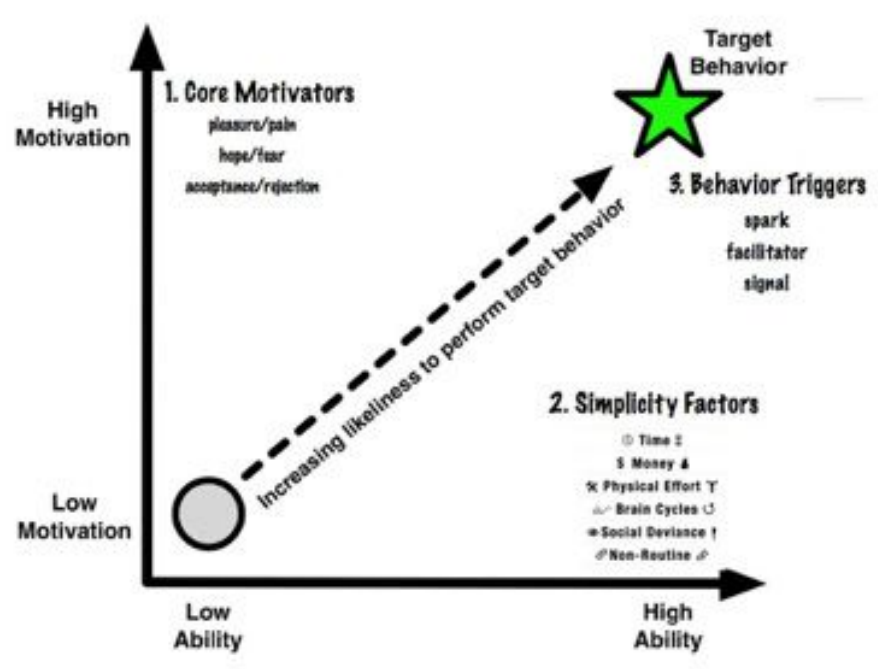

perform such behaviour and be triggered, for example, by a signal. These factors must occur at the same moment, or the behaviour might not happen (Fogg 2009).

Chart: Fogg Behavior Model

Source: Fogg, B. (2009). A Behavior Model

For Persuasive Design

This diagram shows the way the model works. The vertical axis shows the user's motivation, while the horizontal axis shows the user's ability. The third factor is a trigger; without an appropriate trigger, behaviour will not occur even if both motivation and ability are high. Therefore, the higher the levels of the combination of motivation and ability, the more successful a child's response will be to the trigger. In order for the behaviour to happen, the combination of all three circumstances need to be balanced accordingly and the user must have some level of motivation and ability to perform set behaviour change. The task for the designers is to increase the motivation and/or the ability, for example, making the behaviour change simpler by increasing the ability to perform the task. 
The following characteristics need to be considered in order to incorporate a successful trigger: the first is to notice the trigger - this could be a text message, a sound, an announcement, or even a biological trigger such as a growling stomach. Secondly, we associate the trigger with a target behaviour. Thirdly, the trigger coincides with the motivation and ability to perform set task. And lastly, timing, which is often missing in a behaviour change, is considered a key element for persuasion.

According to Fogg, this key element is so important that even ancient Greeks named it Kairos - the opportune moment to persuade (Fogg, 2009). As Fogg (2009) sees it, the opportune moment for behaviour performance is any time motivation and ability put people above the behaviour activation threshold. If we want to perform a behaviour, a well-timed trigger is always welcomed. But when our motivation is low, a trigger is a distraction (p.3).

Research has found that when a task is easy or automatic enough, and there is high motivation, people respond faster to triggers, such as, a red dot on an application, the vibration of a phone, an email marked 'unread' with an inviting subject line. As Fogg says, "[p]ut hot triggers in the path of motivated people" (Fogg, 2009, p.3). Connecting emotionally with the trigger gives the user a rush of dopamine that will motivate the user to engage with the device or application continuously, and eventually form a behavioural change. 


\section{Personalizing Persuasive Technology}

Personalizing an application or device allows the user to customize them to the users' needs and choices. This strategy gives the opportunity to adapt a system's content and functionality accordingly, in order to achieve the intended behavioural change goal. Simulation provides the means of the user to observe the cause-and-effect linkage to their behaviour (Orji, Vassileva, \& Mandryk, 2014). Self or supervised monitoring allows people to track their own behaviour and the change occurring over time, providing information on past and present states.

As mentioned earlier, praising the user for performing the targeted behaviour via messages, images, symbols or sounds is a way to give positive feedback to the user. A points-reward system is often used as "praise" for the tasks achieved towards the desired behaviour change. Competition and comparison are other common tactics utilized to motivate the user. In addition to these strategies, cooperation is commonly used to make the user work together with a team and to achieve their goals collectively (Orji et al., 2014). Hence, tailoring the content and functionality to a particular user's needs allows for a more accurate personalization and identification of the achieved end-goal.

In recent studies, health-care professional and researchers have examined the effectiveness of tailored interventions via personalization. Tailored interventions are often created to imitate an extension of client-to-health-care practitioner or 
counsellor (Kaptein, Markopoulos, De Ruyter, Aarts, 2015). There have also been a number of influence principles suggested in which psychologists apply to increase the effectiveness of their persuasive methods. These principles could also be applied when designing persuasive technology applications. There are six influence principles that support the effectiveness of persuasion (Oinas-Kukkonen, H., and Harjumaa, M., 2009, page\#):

1. Authority: This is considered a form of influence that causes some form obedience and responsibility .

2. Consensus: When the user observes others aligning to the same belief and/or behaviour then he/she is more likely to adopt this behaviour.

3. Consistency and Commitment: People thrive by applying consistency in their beliefs and acts accordingly. This can also be explained in both, attitude and behaviours.

4. Scarcity: This principle increases the perceived value a product or opportunity has. When the opportunity of acting or achieving a goal is limited, this then increases the desire of acting .

5. Liking: When we are "liked" by someone, or we like something, we feel identified with the action and, therefore, act favouring that action.

6. Reciprocity: People are inclined to pay back a favour. This principle, when implemented properly, is exceptionally strong. 
Personalizing the previously mentioned principles leads us to define implicit and explicit profiling. We need these methods, in which we can estimate for each individual user which influence principles will be the most effective (Kaptein, Markopoulos, De Ruyter, Aarts, 2015). Explicit profiling allows us to obtain - using questionnaires - self-reflections of users' own traits. It could also measure the tendencies to certain influence principles, and to adapt the interaction with the user according to the obtained estimates. (Kaptein, Markopoulos, De Ruyter, Aarts, 2015). Implicit profiling uses operative measures to estimate the user's susceptibility to certain influence principles. In applying these principles, it could be argued that all systems that use persuasion profiles should address four key requirements when designing persuasive technology. According to (Kaptein et al., 2015, p. 40) these key systems are:

1. Identification: the ability to identify individual users

2. Presentation: the ability to present the influence principles to users

3. Measurement: the ability to measure a user's traits

4. Single inheritance: the ability to link behavioural observations uniquely in influence principles.

\section{Persuasive Technology in Educational Settings}

The previously mentioned persuasive principles are similarly applied by teachers in their classrooms as educational methods. It is possible to argue that behaviour change and knowledge acquisition are, at the very least, an intertwined process. The 
purpose of teaching is to bring about behaviour and attitude change in domains such as personal and social education, citizenship, and behaviour remediation (Mintz and Aagaard, 2012). Therefore, by implementing these same principles into designing persuasive technology for educational settings, one can reinforce theories like the ones applied in the Fogg Method, such as Kairos.

Fogg (2003) highlights the concept of Kairos - the idea that delivering the right message at the right time is more likely to persuade - and argues that mobile devices and wearable devices are increasingly a central part of the students lives. Mobile technology offers opportunities for learning interventions to be delivered when the student is receptive to receiving them (Mintz, Aagaard, 2012).

Relevant to this subject, there are technologies applied for designing online teaching tools, in which information filtering algorithms make recommendations to users about potential choices. These online tools allow teachers to personalize and tailor specific learning techniques, which allow the teacher and student to have a one-on-one relationship with the subjects taught and the student's personal goals.

Further work by Oinas-Kukkonen and Harjuma, (2009) modified Fogg's original principles and framework, producing more explicit design principles for persuasive systems. It is clear, utilizing their work (showed in table below), how these principles 
could be applied in instructional design for use in school settings (Mintz, Aagaard, 2012). 


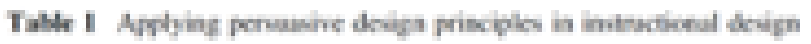

\begin{tabular}{|c|c|c|}
\hline Fistith & IPrroing & 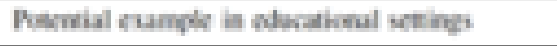 \\
\hline Endetima & 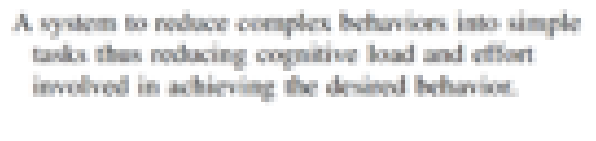 & 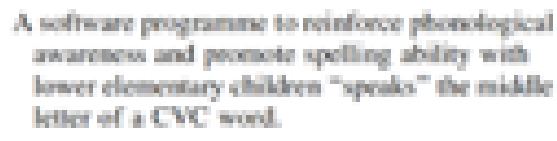 \\
\hline Twaling & 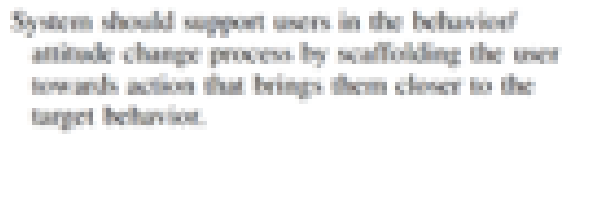 & 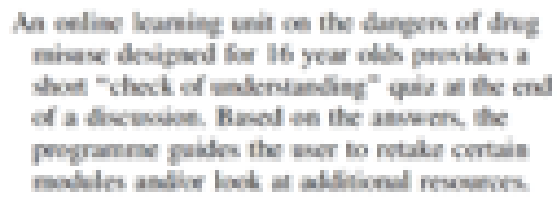 \\
\hline Thiving & 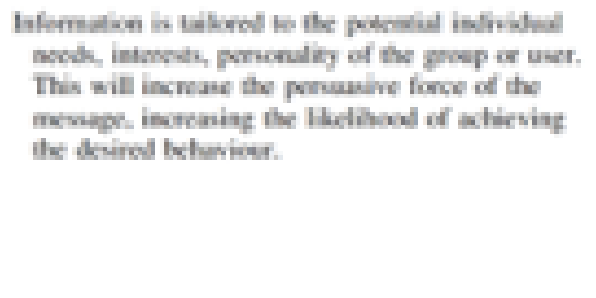 & 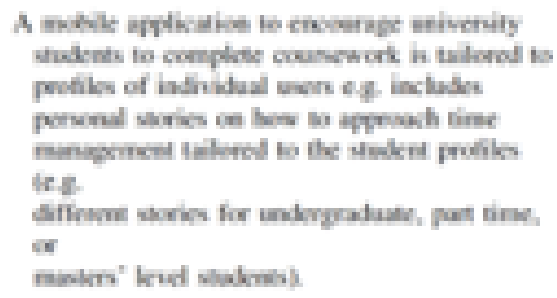 \\
\hline Primoulinaten & 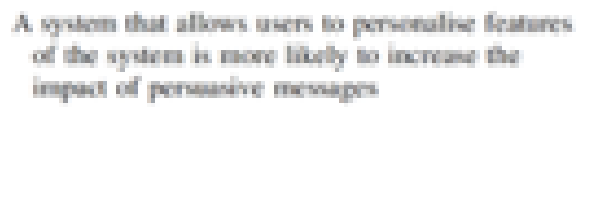 & 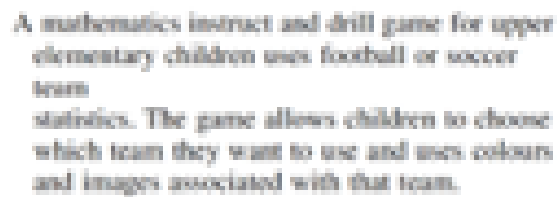 \\
\hline
\end{tabular}

Tubh I antinod

\begin{tabular}{|c|c|c|}
\hline Hishd & 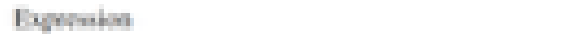 & 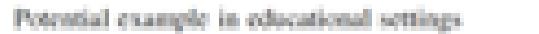 \\
\hline kill rombuts & 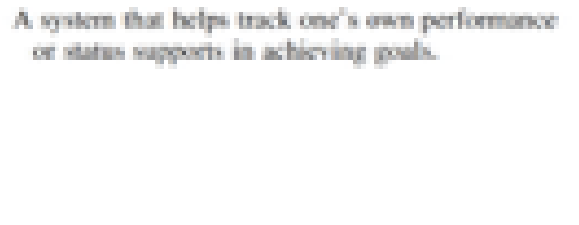 & 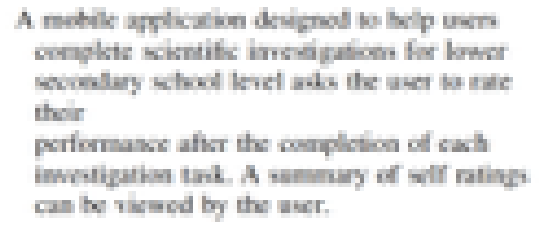 \\
\hline Cindtily & 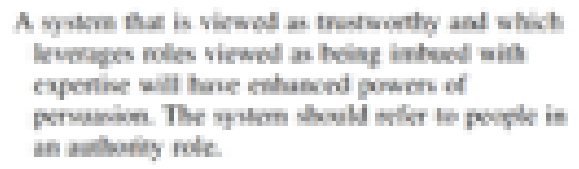 & 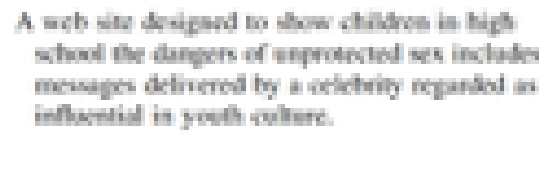 \\
\hline Prsimfire indi & 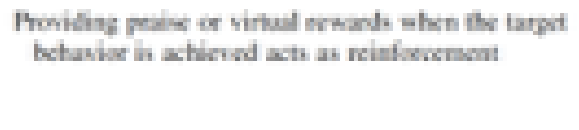 & 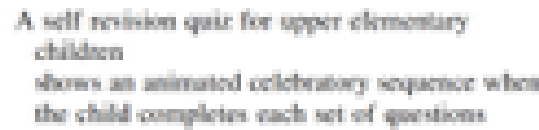 \\
\hline
\end{tabular}

Table: Applying Persuasive Design Principles in Instructional Design

Source: Mintz, J., and Aagaard, M. (2012). The application of persuasive technology to educational settings. Pp. 493-494 
Mintz, Aagaard (2012) argue that credibility is an important design principle for the use of persuasive technology in educational settings because teachers' credibility is an important factor in mediating in classrooms. A number of studies show that there is a relationship between perceived teacher credibility and student behavioural and academic outcomes (Orji, Vassileva, and Mandryk, 2014).

Persuasive design principles could also be successfully applied to other emerging technologies focused on academic learning. Moreover, persuasive design highlights the importance of tailoring (i.e. personalizing), interventions to individual needs profiles. Tailoring is particular relevant for mobile systems, where its combination with the Kairos principle can facilitate the delivery of personalized persuasive study messages at the right time and place (Ferebee, 2010). PT has been researched as a personalized tool for children in educational settings who require a specific level of attention, for example children within the autism spectrum (Mintz, Aagaard, 2012). In addition, this technology has also been introduced in assist mental health conditions such as depression and anxiety disorders, by detecting patterns of anxiety and design a personalized treatment. 


\section{Anxiety Disorder: Selective Mutism}

\section{Definition of Selective Mutism (SM)}

$\mathrm{SM}$, defined earlier in the paper, is a rare anxiety disorder in which children withhold speech in social situations, for example at school or with playmates, despite having the physical capacity to do so, but they speak normally on other situations. Some researchers have suggested that early development risk-factors, such as genetics, histories of immigration, hospitalization, trauma, conflicted family dynamics, and unresolved internal conflict could play a main factor for causing this condition (Hung, Spencer and Dronamraju, 2012).

Children diagnosed within the autism spectrum are often not diagnosed with SM if it occurs as it is a disorder that develops overtime. It can often go unnoticed because it shares many characteristics seen within the autism spectrum and therefore is difficult to link specifically to SM. Other diagnosis might present and led to diagnoses such as: general anxiety disorder, communication disorder, intellectual disability, schizophrenia, mood disorders, as well as others.

\section{Characteristics and Social Challenges of Children Diagnosed with SM}

Some of the characteristics of children with SM have been described as shyness, fear of social embarrassment, withdraw, social isolation, clinging behaviour, temper tantrums, controlling issues, and oppositional behaviour (Hungerford, Edwards and 
lantosca, 2003; Krysanski, 2003). There is a common misconception that SM is a condition that the child outgrows. Selective Mutism occurs in girls more often than boy, according to studies by Hayden, (1980); Wergeland, (1979); Wilkins, (1985). Steinhausen, \& Juzi, (1996) reported a female to male ratio of $1.2-1$. The most commonly reported cases for treatment are for females (Diliberto, Alexandra, 2010). Some research shows that multilingual or bilingual children from an immigrant background are three times more likely to have SM than native children. Rates of SM among immigrant children are for 2.2- 2.8\% (Diliberto, Alexandra, 2010). However, these statistics could be the result of misdiagnosis due to the fact that they refuse to learn a second language (Diliberto, Alexandra, 2010).

Children with SM generally speak at home, but stop speaking at school with their teachers and classmates or in other social environments. Some children might speak with selective friends or classmate but most likely would not speak with adults, except their parents, or other close members of their families. Shyness or mutism might present at home if there are extended members of the family or guests not related to the child. Children with SM rely in other types of communication such as gesturing, signs, nodding, pointing, pushing, writing or grunting. In other cases where children are more verbal, they might use monosyllabic utterances to communicate (Diliberto \& Alexandra, 2010). 
Intervention and diagnosis would ideally occur at an early age. This could be as early as preschool, but symptoms typically become more evident in the early years of elementary school. SM starts presenting as shyness but quickly will show signs of anxiety as well. The child is not able to communicate verbally with his or her teachers or peers, nor are they able to participate in class activities where verbal communication is required, resulting in barriers to educational achievement. The parents are usually not aware of the disorder (Baldwin, Cline, 1991). Although the origins of this disorder vary from study to study (Leonard, Topol, 1993), most of the research indicates that this childhood anxiety is biologically and temperamentally based.

\section{Medical and Psychological Interventions}

Most common interventions used as treatments for SM include behavioural and psychodynamic interventions, medication and multimodal treatments, the latter being the most recommended (Hung, Spencer, and Dronamraju, 2012).

According to Hung, Spencer, and Dronamraju, (2012) the most common interventions, there is:

1. Systematic desensitization: this intervention is also known as "graduated exposure therapy" in which an individual learns to overcome fears in small steps. This would allow the individual to take bigger steps towards self-reliance. 
2. Stimulus fading: also known as "sliding technique". This intervention facilitates a controlled environment where individuals are accompanied by someone they feel comfortable with and slowly adds more people, one person at a time.

3. Shaping: this intervention is also called "vocalizing ladder", and encourages the child to first communicate non-verbally, them make sounds and whisper, and then speak a word, followed by a few words and finally full sentences.

There are cases where medication has to be administered for the treatment of SM. These cases take into account the severity of the disorder as well as the duration and resistance to other intervention methods (Hung, Spencer, and Dronamraju, 2012). Moreover, this resource should always be part of a larger psychological treatment and not used on its own as the sole treatment.

The multimodal approach is another treatment option. This refers to more than one intervention and is the most frequently used approach for the treatment of SM. This approach involves different channels, such as: behavioural interventions, family and/or school participation, in addition to one or more of the following: special education, dance therapy, occupational treatment, and medication (Hung, Spencer, and Dronamraju, 2012). These types of multimodal of treatments have been supported, in the last few years, by digital technology, such as wearable devices and 
other technology that falls in the category of health-ware, or collaborative healthware.

\section{Healthware: Digital Healthcare and Innovation}

Healthware is defined as wearable systems with sensors that continuously monitor the individual's health-related signs, such as: vital signs, sleep patterns, motor activity, body temperature, social interactions, and other health interactions. This device is operated by a software that can use the data collected from these sensors to build a personalized profile of the user's physical performance and nervous system activation throughout the entire day. This provides a more accurate health-related record that can, according to other researchers, give a more accurate medical record (Pentland, 2004).

Understanding face-to-face encounters is critical to developing interfaces that respect and support the wearer's social life. (Pentland, 2004). Social interactions are indicators of mental health and, therefore, an imminent challenge is to build technology that can predict social patterns and dynamics, thereby resulting in the ability to predict mental health disorders.

Most recent diagnoses are made by the population average rather than the patients individual characteristics, which allows for a considerable margin of error. With this data-poor system, it's usually a trial-and-error method that allows the doctor to give 
the correct doses to the patient. It is frequently either over- or underestimates and unforeseen drug interactions commonly occur (Pentland, 2004). The goal of Healthware is to provide effective monitoring of medical treatments. As new and improved sensors become available, and computing technology improves, healthcare professionals are able to organize significant amounts of medical data and can track patients' medical history, including every test taken and medication prescribed over their patients' lifetimes. In addition, this data can provide epidemiological information for use in improving medical knowledge and as a result keeping healthcare costs low. What can make affordable, wearable technology a real asset for health care? One option is to create a simple regulatory framework that doesn't suppress innovation but helps wearable devices become validated in the context of their health-oriented value (Piwek, Ellis, Andrews, Joinson, 2016).

The best approach to make healthware technology viable and affordable is to involve all parties that benefit from this technology. If this technology benefits not just the patient and their diagnosis, but also their families, along with the physicians and the healthcare system, this resource will become a collaborative, inclusive practice.

\section{Collaborative Healthware}

Collaborative healthware is the application of information and communication technologies designed to enhance decision-making and communication between providers, patients, and their families (Lewis, Chang, and Friedman, 2005). This 
popular emerging technology will play an important supporting role in the relationship between the patient and health provider, and will improve the understanding of their illness and how their values affect their decision-making and therefore, treatment procedures.

Collaborative healthware, is defined as "software for healthcare", tightly integrated with people systems (Lewis, Chang, and Friedman, 2005). It is a creative and successful implementation that empowers the patient in their care and care-management (Lewis, Chang, and Friedman, 2005). Since effective healthcare requires communication among many parties, consultation and collaboration among colleagues, as well as with their patients, their families and community resources, shouldn't technology applied to healthcare facilitate this tasks?

\section{Advantages and Challenges of Collaborative Healthware}

Researchers state that collaborative healthware could become a "secondary" diagnostics tool (Piwek, Ellis, Andrews, Joinson, 2016). Wearables could effortlessly provide detailed data on patients with crunch conditions without involving sophisticated, uncomfortable and expensive alternatives. For example, in patients with mental health issues such as depression, a wearable could determine the severity of the symptom base on the number of social interactions, physical activity and sleep patterns, using a wristband and/or smartphone application. One advantage is that wearables could resolve some of the limitations of other 
interventions by providing instant and individualized feedback, giving the patient the power to acknowledge their own state and act accordingly to the response.

A clear challenge is how the development of wearables exist in a "grey area" regarding user safety. The data generated by these wearables could compromise patients' and medical practitioners' privacy, which could be problematic and ethically questionable. People that buy wearable technology today, do not "own" the data generated by their use (Piwek, Ellis, Andrews, and Joinson, 2016). Often the data is collected and stored by the manufacturer, and this could become a risk in the user's information confidentiality.

Another challenge is the reliability and behaviour usability of the decoded data. Currently the feedback given by these devices is based on simple descriptive statistics, for example: average calorie burn rate, weekly heart rate and level of activity. Simple summaries of statistics appear almost trivial given the complex nature of the data that most wearables collect. The same criticism can also be applied to the sociodemographic information recorded by users. The next step will be to move from unsophisticated exploratory feedback to intelligent and personalized explanatory feedback (Piwek, Ellis, Andrews, and Joinson, 2016). There is still a long way to go before wearable technology is satisfactorily accurate and reliable. 
Healthware as aid on treatment for children

There are numerous ways to apply technology in paediatric treatments. The diagram below illustrates the framework in which the application of technologies in clinical care and research with children with chronic conditions increased parent satisfaction (Siarkowski-Amer, 2006). 

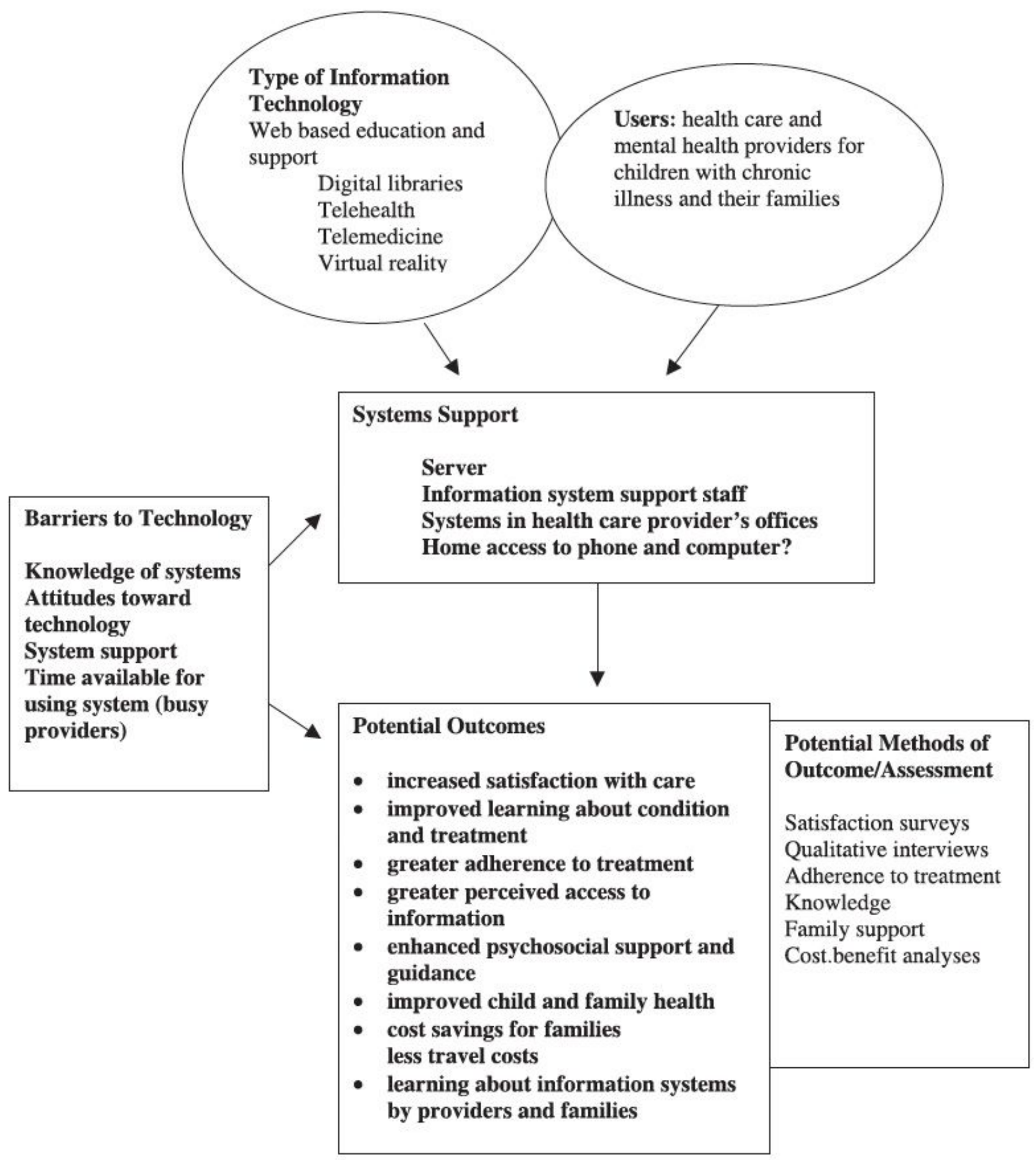

\footnotetext{
Diagram 2: Conceptual Framework to Describe the Application of Technologies in Clinical Care and Research with Children with Chronic Conditions

Source: Siarkowski-Amer, K. (2006). Innovations in Pediatric Health Care Technology: A Multidisciplinary Conceptual

Framework for Using and Evaluating Information Systems. Pp. 3-9
} 
Paediatric healthcare is taking an innovative direction by evaluating the outcomes of the use of technology. Siarkowski-Amer (2006) states that

[t]he broad array of potential outcomes of the use of technology encompass patient care outcomes, provider-based outcomes (e.g., accuracy and ease of diagnosis), and treatment efficacy...(p. 5)

Although this evaluation is heading into a possible positive outcome, there are a number of special related issues that could affect the result of this evaluation in paediatric patients with chronic illness, such as diverse physiologic, psychological, and social situations, the uniqueness of each family must be included in the evaluation of technology use (Siarkowski-Amer, 2006).

In conclusion, a new age of technology has opened up many possibilities to enhance both provider and patient satisfaction and efficiency. The conceptual model presented here illustrates the need for a hub of information with backed-up supports systems and an adequate knowledge-base for families and healthcare providers (Siarkowski-Amer, 2006). In addition, Siarkowski-Amer (2006) advises that the benefits, barriers, and outcomes shown on this conceptual model need to be consistently evaluated over time so that the best available technology to accomplish the best benefit is used. 


\section{Chapter 3: Analysis, Methodology and Implementation}

Based on the growing advances in persuasive technology, as well as in the research done on collaborative healthware, there is an increasing growth in the creation of supporting tools in the treatment of anxiety disorders. Specifically focusing in children diagnosed with SM, this approach could benefit the outcome of their treatment, not just for the patient and their families, but also for the medical institutions.

In regards to the desired behaviour change, the goal is suggesting a system using The Fogg Method and Fogg's predecessors, by motivating a child to complete small tasks, reaching milestones, and getting rewards resulting in creating a change in the child's behaviour. In this case, the desired change would be teaching the child coping mechanisms to use in moments of anxiety and thereby helping the patient gain confidence in speaking in routine circumstances. These goals could be achieved by adapting a persuasive technology model to psychological interventions such as, systematic desensitization, stimulus fading, and shaping (Hung. Spencer, and Dronamraju, 2012).

In addition, the purpose of this research is to investigate the impact of collaborative healthware technology, in supporting therapists and caregivers in understanding the behavioural patterns and changes of the child during the time of the treatment. Moreover, with the results from the data collected by this technology, healthcare 
providers could have a more informed action plan that will allow them to personalize a treatment plan.

\section{Analysis: Selective Mutism and Collaborative Healthware}

Children diagnosed with selective mutism are often presented as quiet and shy individuals. They often speak and are social individuals at home and with close relatives, while at school and other social situations are non-verbal, quiet and reserved. They tend to stay close by their parents and/or other relatives in unfamiliar social situations, like birthday parties, playdates and outings in open spaces. At school, selectively mute children sometimes present themselves as non-verbal, strong-willed, avoidant and irritable students. Some teachers and parents misinterpret these behaviours, especially avoidance, as a controlling or oppositional instead of a manifestation of social anxiety (Diliberto \& Alexandra, 2010).

In order to treat children with SM, therapists and physicians rely in the parents' input to establish a relationship with the child. Moreover, often times teachers and school principals, special education assistants and language therapists are also involved in the treatment. Hence, it is important to have good communication channels among all the parties in charge of the child's treatment. By relying in digital technology, such as collaborative healthware, better methods of communication and collaboration between all the parties involved could be facilitated. This technology - not restricted to wearables but could include other channels such as: data collection and sharing, 
machine learning and even virtual messaging or chats - could open other ways of collaborating, and targeting specific needs of the child, as well as implementing more accurate methods of treatment.

In regards of the behaviour change, by implementing a persuasive technology designed software into the healthware and personalizing the treatment, the patient and their caretakers would be able to monitor the progress in the behaviour change and make adjustments accordingly. This change might be successfully achieved by "gamifying" the interaction of the child with the treatment.

Gamification provides the means to increase an individual's (e.g., consumers, patients, healthcare workers) fun, engagement and compliance, while accomplishing wellness and/or healthcare activities, with positive results on the healthy outcomes and costs of services. The potential applications of gamification are considerable. Through the use of a game-like approach, game elements such as play, fun, challenges, rules, transparency and rewards, can be addressed to almost any real-world problem. (Pereira, Pedro, Duarte, Emília, Rebelo, Francisco, Noriega, Paulo, 2014, p. 753)

\footnotetext{
Methodology: Behaviour change by implementing the Fogg Method, resulting in teaching anxiety coping mechanisms to selectively mute children

In order to implement this method efficiently, it needs to take into consideration several characteristics of persuasive technology. Firstly, it's recommended to follow
} 
a behaviour change support system (Oinas-Kukkonen, Harri and Harjuaa, Marja, 2009), where the patient and all stakeholders need to be aware of a desired behaviour change. This method should be consistent and predictable; for example: giving the patient and their caregivers the opportunity to set achievable goals and analyze the outcome and review them at the end of a session. Personalization is a fundamental characteristic for PT to succeed. Allowing the patient and their caregivers to tailor the actions, goals and outcomes programmed to the healthware device, aligning them with the medical treatment with consistency, commitment and authority, which are main principles that support the effectiveness of persuasive technology.

According to Fogg, this model is based on the fundamentals of learning to automate behaviour-change (2009). There are three basic factors in which this model is based: motivation, ability and triggers. In order for the model to be effective, the combination of all three circumstances needs to be balanced accordingly. The child must have some kind of motivation and ability to perform a behaviour change, sometimes increasing the motivation and/or the ability, makes the behaviour change simpler and more effective.

A strong motivator for children in general, but specifically while dealing anxiety disorders, is praise. This could be a very effective method because it takes the attention away from the challenge and focuses it on what the approval and 
recognition of their parents or the program itself. When the child feels safe and recognized, it's easy to keep them motivated in taking the next step towards finishing the treatment, and to keep working towards the goals determined by the physician and therapists.

Moreover, timing is in the essence of PT applied with The Fogg Method. Based on this method, Ferebee, (2010) describes Kairos, as delivering the right message at the right time. This means that when teaching a child with SM how to manage their anxiety and communicate verbally, the triggers and/or messages should be delivered at the right time, when they are needed. This would facilitate, not only of the emotional response of the child, but also he/she will learn to identify those moments where their anxiety rises, and how to control it.

In the specific case of children with SM, the ability to complete a task might be compromised by the anxiety disorder itself, which is why it is important to highlight that this technology should be a support tool of the medical treatment and not the main source of treatment. The goal is for the child to learn how to manage their anxiety in specific situations and to acquire verbal communication skills to be applied in their daily life, for example at school or other social situations. This thesis proposes a method in how to automate this behaviour. A common scenario for applying the Fogg Method could be when the child is immersed in an educational 
setting, for example at during school hours, or extracurricular activities, training sports, or something similar.

\section{Implementation}

Before a diagnosis is made in a child with $\mathrm{SM}$, he or she would have shown signs that could have caused the parents and/or teachers to seek medical help, for example, extreme shyness and non-verbal skills during school hours. After the diagnosis is confirmed, parents and medical experts choose a course of action. This action consists of a series of psychological interventions and sometimes combined with medication if necessary (Hung, Spencer, and Dronamraju, 2012).

When applying Oinas-Kukkonen and Harjuma (2008) principles to persuasion, such as consistency and authority, combined with the Fogg's method, teach children with SM how to cope with anxiety in daily situations. This behavioural framework is determined by personalizing the immediate and long term goals in the patient's treatment. Based on the parameters established by the physicians and therapists, the parents or caregivers and child could follow a progression of small steps, also known as systematic desensitization, to accomplish learning how to cope with anxiety and start gaining verbal skills as a final goal.

This method could also be based on a praise/reward system, in which the child/patient will get immediate positive messaging and, when completing small 
tasks, could gain points towards bigger rewards. These rewards could be determined by the physician and parents combined. For example, if the child wants a much desired toy or an outing, the points system could show-on a device or computer interface- how many more tasks needed to be completed in order to get the reward. By "gamifying" the interaction of the child with the PT interface, the progression of the treatment might be seamless to the child and he/she will learn the desired skills without feeling that it is a chore or an obligation.

This personalized treatment could be housed in a PT software system, which will include not only a smartphone or wearable interface but also a computer interface that could allow all the involved individuals to access the information gathered by this PT system. For example, if the child uses a wearable device, the biometrics could be gathered and reported back to the parents and physician. As well, the "gamified" treatment could allow the child to have a more immediate and holistic response, giving all the parties involve a more complete picture of the child's progress.

In conclusion, this major research paper investigates the understanding of the role of persuasive technology in the implementation of alternative digital platforms to support mental health treatments in children, specifically those related to SM. Moreover, it has aimed to analyze the effectiveness in the behavioural change by adding timely trigger functions integrated into a wearable device. And at the same 
time, to understand how this technology help caregivers manage treatment applying it to collaborative healthware. 


\section{Chapter 4: Case Studies}

For the last several years, there has been various innovating minds in the technological industry that have developed or are currently developing similar assistive technology systems. These systems have different functions and focus their efforts on integrating not just persuasive technology but machine learning frameworks within the healthcare industry.

So far, none of the cases researched for this thesis touched on treatments for SM specifically. There are one or two assistive technology initiatives focused on children within the Autism Spectrum such as "Revel", by Awake Labs (awakelabs.com, 2016), which collects data via bio-sensors on a wearable device and gives a precise reading of the child's biometrics. The information gathered by these sensors gives the therapist a baseline of data that allows for a better understanding of regular anxious patterns and social triggers.

Another initiative is "The Hands Project (Helping Autism Diagnosed young people Navigate and Develop Socially)," which is a multi-disciplinary project that has developed a mobile software solution based on persuasive technology to help young people (aged 11-16) with high-functioning Autism Spectrum Disorders (ASD) to become better integrated into society (Mintz \& Aagaard, 2012). 
Moreover, Aparito, a wearable/application system that tracks medical treatment in children diagnosed with rare diseases, by tracking data and using collaborative healthware. This device gives a more holistic approach for treating these diseases and enables long-distance monitoring on the patient's treatment and daily life routines. In addition, there are applications created to gamify behaviour-change using Role Playing Games (RPG). RPG, a game in which players take on the roles of imaginary characters who engage in adventures, typically in a particular computerized fantasy setting overseen by a referee (Oxford Dictionary, n.d). 'Habitica' (2013) is an example of RPG applied in persuasive technology, in which players are motivated to adopt positive habits by completing personalized tasks via an application and/or website portal and at the same time by collaborating with peers thereby becoming more socially accountable.

The case studies presented below are examples of how applications based on persuasive technology and collaborative healthware are creating a more inclusive environment for improving healthcare and personal well-being.

\section{Reveal, by Awake Labs}

'Reveal' is a wearable technology created by Awake Labs, a company founded by Andrea Palmer, CEO and Paul Fijal, CPO, both Mechanical Engineers from the University of British Columbia. This device works by measuring three major indicators of anxiety - heart rate, temperature and electrodermal activity (also known as "emotional 
sweat") - through a pair of electrodes embedded in the band.

Those signals are transmitted to the Reveal smartphone app, notifying parents and caretakers of impending 'behaviour meltdowns,' so preventative measures can be taken. Caregivers can also add contextual data to help the app develop a complete profile of the child. (awakelabs.com, 2016).

This device is designed to measure and track body's responses in real-time to better understand triggers and behaviour patterns. This measurement helps to predict reactions to certain situations. Many people on the spectrum have specific triggers that can lead to overstimulation and rises in anxiety. 'Reveal' helps children within the Autism Spectrum that have difficulty with verbal communications, social interaction, as well as relating to their own emotions (awakelabs.com, 2016).

Every individual within the autism spectrum is unique, but many people within the spectrum have difficulty communicating, and it often results in behavioural meltdowns. These meltdowns are emotionally draining and could be physically and emotionally dangerous, not just for the individual, but for the parents and caregivers as well. 'Reveal' helps in predicting this meltdowns, therefore, the caregiver can help reducing the anxiety before the meltdown occurs. This alert is given to the caregiver via a smartphone application to help intervene at the right time. 


\section{The HANDS Project: Helping Autism Diagnosed young people Navigate and Develop Socially}

'HANDS' is a mobile application that helps young people, age 11-16, within the Autism Spectrum to become better integrated into society by supporting them to develop social skills and self-managements skills. This application uses a user-centric design within collaborative healthware principles to integrate the needs of the child, their parents and teachers in accomplishing the latter goals. The application consists of a web-based toolkit that teachers use to develop support and intervention sequences specific to the needs of each child. These sequences consist of a series of screens, each of which can include personalized texts, images and videos referred as "personal trainer" sequence.

This framework has been very successful in scenarios wherein the child has strong and clear motivations to achieve the targeted behaviour-change. This attitude facilitates the teacher to be able to use HANDS as a toolkit on mobiles applications, such as daily prompts for desired behaviour change, and also provides more face-to-face interaction between the teacher and the student that matches the desired goals (Mintz, Aagaard, 2012). The user testing of this application with teachers brought unanimous feedback indicating that HANDS was only successful when this type of technology is integrated within an existing teacher-child relationship (Mintz, Aagaard, 2012). 


\section{Aparito}

Another device with a similar approach to 'Reveal' is 'Aparito' (www.aparito.com, 2017). Aparito is a wearable device and a collaborative healthware application that provides remote patient monitoring outside the hospital environment to patients diagnosed with rare diseases submitted to trial research. This technology was developed in London, UK by Elin Haf Davies, a Medical Scientists from the University of London, UK.

This divide collects data from patients in real-time and delivers it to their physicians. 'Aparito' connects doctors and patients, as well as their caregivers/parents via remote monitoring, improving the understanding of the disease and the clinical trial outcomes. A particular area of expertise is the evolution of orphan drugs for rare diseases. Orphan drugs are defined a drug that is not developed or marketed because its extremely limited use makes it unprofitable (Webster dictionary n.d), and it's manufactured to treat the conditions referred to as an orphan disease, which is defined as a disease that affects a very small percentage of a population (Webster dictionary n.d). This technology is suited to all ages of patients from small children to elderly individuals. This wearable device platform uses Android and iOS applications in conjunction with a core system built around enterprise class middleware delivered from one of Aparito's partners data centres, (Microsoft Azure and Amazon EC2). Alternatively, an on-premise solution can also be delivered if required (www.aparito.com, 2017). 


\section{Habitica: Habit RPG (Role Player Game)}

'Habitica' RPG is a video game to help improve real life habits. It "gamifies" the user's behaviour change by turning all their daily tasks (habits, dailies, and to-dos) into little monsters that have to be conquered. The more engaged the user is in the game, and how accountable they are to change habits, the more motivation they have to keep improving in their goals. As a result the user gets rewards such as coins that allow the user to buy swords or other weapons to defeat the monsters. If the user loses motivation, the characters start "backsliding" in the game (https://habitica.com/static/features).

Tyler Renelle, the creator of this RPG, originally wanted to help change his own habits after being inspired by the books, The Power of Habit and The Now Habit. His first version was created as a colour-coded Google Docs coded cell formulae. As the popularity of the tool grew, Renelle reached out to Siena Leslie and Vicky Hsu to help developing a more complex version of Habit RPG, later on becoming co-founders of Habitica. (en.wikipedia.org/wiki/Habitica). This is a community based development app, where the members of the Habitica community help improving the application, for example by creating pixel art, translating text, creating music and sound effects, and promoting the app in other online communities. 
The application became more popular when Renelle started a Kickstarter campaign in January of 2013 , which allowed him to develop a more complex application. This app/game is designed to help the user improve his/her habits by setting goals and reaching milestones while role playing a personalized character or avatar. When the goals are reached, the user gets an armour or a weapon to add to his/her collection and to fight monsters. This is based on a model of motivation, consequences, accountability, and instant gratification. This application is widely used in reaching educational goals and improving good habits.

The game consists of achieving different levels, and depending on how the user completes their to-dos and "dailies" is the amount of power or "health" they get. The more habits they complete the more health they get, and if they stop playing the characters dies. The currency of the game is also based on the accumulated "health" or habit completion, the user gains gold and/or silver depending on the difficulty of the task. This currency allows the player to buy items — within the game- such as eggs, colour potions or food. Additional pets and mounts can be obtained during special events and by completing quests.

(en.wikipedia.org/wiki/Habitica). Because this is a community based game, the social accountability aspect is essential. Users can compare and compete with other players with similar goals, for example, exercising everyday, also can join "guilds" and participate in challenges made by other players. Users can also collaborate with other players and focus on accountability. 
This application's programming interface (API) allows programmers to create third-party applications extensions and other tools that interface with Habitica. This functionality allows the players to extend the game to achieve personal long term goals.

All the above mentioned initiatives, were clearly developed after specific needs and a lack of resources to satisfied them. They also have had an impact in collaborative healthware research, while technological advances for healthcare and personal well-being have occurred. The specific cases focusing in children have a major influence in the research of this thesis. 


\section{Chapter 5: Conclusion}

The use of digital technology in the healthcare industry has become more immersed in patient treatment and therapy-development, hence the need to understand the advantages and disadvantages of its use. Persuasive technology applied in behaviour change - particularly in shaping behavioural changes in children - needs to be moderated and screened by consensus. This consensus is fundamental in the understanding and consistency of the goals established by all parties involved and in its outcome. Therefore, tailoring the content and functionality to a particular user's need, based on their specific needs, allows a more accurate personalization and identification of the achieved end-goal.

Focusing these efforts in treating children diagnosed with SM specifically, this type of technology could be applied in educational settings, as this is the environment where the child struggles most significantly with this disorder. A child with $\mathrm{SM}$, holds his/her speech in situations or environments outside their homes, therefore, therapists, teachers and other school professionals, and parents all working collaboratively in implementing a consistent treatment, would allow for much better results.

Integrating persuasive technology to any medical treatment has a number of advantages and disadvantages. Focusing on treatment for children, especially with regard to behavioural change, this type of technology needs to be regulated in terms 
of ethical norms and privacy. In regards to medical data collection and privacy, the system designed to collect this information requires a strong understanding of the data collected, as well as the implications of the distribution of the patient's personal information.

While this is the argument of this paper, the author also acknowledges that more research is needed to corroborate the effectiveness of this proposal. In terms of involving children with SM, there are several factors to consider such as stubbornness, shyness and unwillingness to participate in such an approach to treatment. Therefore, creating appropriate systems is fundamental. Prototyping and designing a series of interfaces that would allow easy personalization, then implementing a rapid prototyping and testing is recommended. Moreover, testing this prototype specifically with children undergoing treatment, as well as, consulting with the parents and physicians involved in such treatment is fundamental for obtaining accurate feedback.

In conclusion, the primary reason for this proposal followed by steps mentioned above, is to create an alternative aid that will enhance the quality of life for children with SM. Moreover, teaching not just the child, but their family, to understand and manage this anxiety disorder will allow them to become more independent individuals. 


\section{References}

Aparito: Wearable technology and mobile apps for disease monitoring. (n.d.). Retrieved August 26, 2017, from https://www.aparito.com/

Reveal by Awake Labs. (n.d.). Retrieved August 26, 2017, from http://www.awakelabs.com/

Diliberto, R. A. (2014). Oppositional And Anxiety Behavior Profiles In A Clinical Sample Of Youth With Selective Mutism By Rachele Alexandra Diliberto Bachelor of Arts in Psychology Arizona State University $A$ thesis submitted in partial fulfillment of the requirements for the Masters in Psychology.

Habitica. (2017, June 10). Retrieved August 26, 2017, from https://en.wikipedia.org/wiki/Habitica

Ferebee, S. S. (2010). Successful persuasive technology for behavior reduction: Mapping to Fogg's Gray Behavior Grid. In Lecture Notes in Computer Science (including subseries Lecture Notes in Artificial Intelligence and Lecture Notes in Bioinformatics)(Vol. 6137 LNCS, pp. 70-81). https://doi.org/10.1007/978-3-642-13226-1 9

Fogg, B. J. (2003). Computers as Persuasive Social Actors. Persuasive Technology: Using Computers to Change What We Think and Do, 89-120. https://doi.org/10.1145/764008.763957

Fogg, B. (2009). A behavior model for persuasive design. In Proceedings of the 4th International Conference on Persuasive Technology - Persuasive (pp. 40-46).

ACM.https://doi.org/10.1145/1541948.1541999

Improve Your Habits by Playing a Game. (n.d.). Retrieved August 26, 2017, from https://habitica.com/static/features

Hung, S.-L., Spencer, M. S., and Dronamraju, R. (2012). Selective Mutism: Practice and Intervention Strategies for Children. Children and Schools, 34(4), 222-230. https://doi.org/10.1093/cs/cds006

Kaptein, M., Markopoulos, P., De Ruyter, B., and Aarts, E. (2015). Personalizing persuasive technologies: Explicit and implicit personalization using persuasion profiles. International Journal of Human Computer Studies, 77, 38-51. https://doi.org/10.1016/j.ijhcs.2015.01.004

Lewis, D., Chang, B. L., and Friedman, C. P. (2005). Consumer Health Informatics. In Consumer Health Informatics: Informing Consumers and Improving Health Care (pp. 1-7). Retrieved from http://books.google.com/books?id=y6S_K7U 3w8C\&pgis=1

Long, S., Spurlock, D. G., Fjeldsoe, B. S., Marshall, A. L., Miller, Y. D., Noell, J., Steiner, J. F. (2013). Interactive Technology Applications for Behavioral Counseling: Issues and Opportunities for Health Care Settings. American Journal of Preventive Medicine, 20, 80-7.

https://doi.org/10.1016/j.amepre.2008.09.040

Mintz, J., and Aagaard, M. (2012). The application of persuasive technology to educational settings. Educational Technology Research and Development, 60(3), 483-499.

https://doi.org/10.1007/s11423-012-9232-y 
Oinas-Kukkonen, H., and Harjumaa, M. (2009). Persuasive Systems Design: Key Issues, Process Model, and System Features. Communications of the Association for Inf. Sys., 24(1), 485-500.

Retrieved from

http://search.ebscohost.com/login.aspx?direct=true\&db=bth\&AN=45267822\&site=ehost-live

Orji, R., Vassileva, J., and Mandryk, R. L. (2014). Modeling the efficacy of persuasive strategies for different gamer types in serious games for health. User Modeling and User-Adapted Interaction, 24(5), 453-498. https://doi.org/10.1007/s11257-014-9149-8

Pereira, P., Duarte, E., Rebelo, F., and Noriega, P. (2014). A review of gamification for health-related contexts. In Lecture Notes in Computer Science (including subseries Lecture Notes in Artificial Intelligence and Lecture Notes in Bioinformatics) (Vol. 8518 LNCS, pp. 742-753). Springer Verlag. https://doi.org/10.1007/978-3-319-07626-3 70

Piwek, L., Ellis, D. A., Andrews, S., and Joinson, A. (2016). The Rise of Consumer Health Wearables: Promises and Barriers. PLoS Medicine, 13(2). https://doi.org/10.1371/journal.pmed.1001953

Pentland, A. (2004). Healthwear: medical technology becomes wearable. Computer, 37(5), 42-49. doi:10.1109/mc.2004.1297238

Siarkowski Amer, K. (2006). Innovations in Pediatric Health Care Technology: A Multidisciplinary Conceptual Framework for Using and Evaluating Information Systems. Children's Health Care, 35(1), 5-10. https://doi.org/10.1207/s15326888chc3501_2 